\title{
Highly Fluorescent Molecularly Insulated Perylene Diimides: Effect of Concentration on Photophysical Properties
}

\author{
Bolong Zhang, Hamid Soleimaninejad, David J. Jones, Jonathan M. White, Kenneth P. Ghiggino, \\ Trevor A. Smith and Wallace W. H. Wong*
}

School of Chemistry, Bioz1 Institute, The University of Melbourne, 30 Flemington Road, Parkville, Victoria, 3010, Australia.

\begin{abstract}
A series of four perylene diimide (PDI) chromophores were prepared with increasing steric bulk on the imide substituents with the aim of retarding the effect of concentration quenching on photoluminescence, commonly observed with these dyes. Spectroscopic investigations of the compounds in dilute solution confirmed that the photophysical properties of the PDI core chromophore were not perturbed by the bulky substituents. Solid film samples containing the PDI compounds at various concentrations dispersed in a poly(methyl methacrylate) (PMMA) matrix were examined and compared to amorphous neat films as well as crystalline samples. The PDI compounds containing di-tert-butylphenyl (bPDI-3) and trityl (bPDI-4) substituents showed near unity photoluminescence quantum yield (PLQY) up to $20 \mathrm{mM}$ in PMMA compared to $10 \%$ PLQY for the reference compound (bPDI-1) without molecular insulation. Surprisingly, high concentrations $(>40 \mathrm{mM}$ ) of a phenyl substituted PDI compound (bPDI-2) with moderate molecular insulation formed emissive aggregates that showed a higher PLQY compared to the PDI derivatives with greater steric bulk. By examining the molecular structure and solid state packing in conjunction with a series of photophysical measurements, new insights into designing highly fluorescent dyes, particularly in the solid state, were obtained. The trityl substituted PDI compound (bPDI-4) was used in a luminescent solar concentrator with optical quantum efficiency of 54\%, flux gain of 6.4 and geometric gain of 45 .
\end{abstract}

\section{Introduction}

Perylene diimide (PDI) is a polycyclic aromatic chromophore widely used in the dye industry and has been studied as an advanced material with applications in fluorescence labelling, organic semiconducting devices ${ }^{2,3}$ and light harvesting., 5 The PDI chromophore can be easily functionalised to achieve derivatives with desired properties including the tuning of photon absorption and emission while maintaining good photo- and thermal stability., 6, 7 One key feature of PDI compounds is that their conjugated perylene core has a strong tendency to intermolecular $\pi-\pi$ stack leading to molecular aggregates. While these aggregates or supramolecular assemblies can lead to desirable materials properties, such as charge transport, photophysical properties of the PDI chromophore can also change significantly. ${ }^{8-10}$ Apart from perturbation of the absorption spectrum, a commonly observed characteristic of PDI chromophores is the decrease in photoluminescence quantum yield (PLQY) with increasing concentration. ${ }^{11}$ Clearly, this observation is a result of the formation of $\pi-\pi$ stacked PDI dimers and/or larger aggregates. By preventing $\pi-\pi$ interactions between PDI molecules, it is possible to achieve high PLQY at elevated concentrations. ${ }^{12}$ The primary goal of this study is to determine the limits of PLQY in passing from dilute solution to the solid state of appropriately designed PDI chromophores.

There are two main approaches to prevent the $\pi-\pi$ stacking in PDI chromophores. One method is to install sub- stituents at the bay-region (1, 6, 7, 12-positions) of the perylene core to impart a twist in the normally planar conformation (Figure 1a). ${ }^{6}$ By doing so, the tendency of $\pi$ $\pi$ stacking and intermolecular association is reduced, but not totally eliminated. Bay substitution also significantly alters the photophysical characteristics of the PDI chromophore which may or may not be desirable depending on intended applications. ${ }^{13-15}$ Molecular insulation is the other effective approach to reduce the intermolecular $\pi-\pi$ stacking effect in PDI compounds. ${ }^{12}$ By positioning substituents (or molecules) around the planar perylene core, one can sterically block $\pi-\pi$ stacking preventing chromophore-chromophore interactions. ${ }^{16,17}$ With this approach, it is possible to eliminate $\pi-\pi$ stacking association between PDI molecules while maintaining the original photophysical characteristics of the individual PDI chromophore.

Covalent substitution is possible through the shoulder (2, $5,8,11$-positions) of the perylene or through the imides without disturbing the planarity of the PDI core (Figure 1a) ${ }^{18,19}$ However, substitution at the shoulder position has a significant impact on the optical and electronic properties of the PDI chromophore. ${ }^{18,20}$ As it is important to maintain the original photophysical characteristics of the individual PDI chromophore for our application, vide infra, we decided to substitute through the imide positions. It should be noted that it is also possible to achieve supramolecular insulation with one example using cucurbit[8]uril to encapsulate a PDI molecule in water. ${ }^{21}$ The 
PDI-cucurbit[8]uril host-guest association was driven by hydrophobic-hydrophilic interactions in an appropriate solvent environment.

Substituting via the imide positions leaves the perylene core untouched $5,{ }^{22}$ and is synthetically more versatile, enabling the creation of a series of compounds. One of our previous articles reported a molecularly-insulated PDI molecule with four di-tert-butylphenyl groups substituted via the imide position, labelled as bPDI-3 in this work (Figure 1d). ${ }^{22}$ This molecule showed near quantitative PLQY in dilute conditions and close to $75 \%$ PLQY at a higher concentration $(80 \mathrm{mM})$ dispersed in a thin-film poly(methyl methacrylate) (PMMA) matrix. With such a high PLQY at high concentration, this molecularlyinsulated PDI was used as a fluorophore in luminescent solar concentrator (LSC) devices taking advantage of an energy migration and energy trapping strategy (vide infra). ${ }^{2,2}, 23,24$

In this article, four PDI derivatives (bPDI-1, $-2,-3$, and -4; Figure 1) were synthesized (see Supporting Information for details) with the purpose of studying the correlation between the size of imide moeities and the photoluminescence quantum efficiency at high concentration. The UV-vis absorption and photoluminescence (PL) spectra of the PDI samples were recorded in both solution and solid state under a range of concentrations. The effects of the bulky substituents on the aggregation behaviour of the PDI molecules are discussed with reference to the crystallographic data. Absolute PLQYs of PDI molecules dispersed in the PMMA matrix were measured using an integrating sphere to observe the effect of the substituents on photoluminescence quantum efficiency. Finally, the trityl-substituted bPDI-4 was tested in light harvesting LSC devices as a Förster resonance energy transfer (FRET) donor. In comparison with the previously reported bPDI3 system, ${ }^{12}$ the fully molecularly-insulated bPDI-4 demonstrated improvements in both PLQY and performance in LSC devices.

\section{Photophysical properties of PDI derivatives}

The UV-vis absorption spectra of all four PDI compounds (bPDI-1 to bPDI-4) in dilute solution $\left(2.5 \times 10^{-5} \mathrm{M}\right.$ in $\mathrm{CHCl}_{3}$ ) are identical to each other (Figure 2). The PL spectrum of the PDI compounds are also identical in these dilute solution samples. As anticipated, the imide substituents in this compound series did not perturb the photophysical characteristics of the PDI chromophore (i.e. the substituents had no significant conformation or electronic influence). This was further confirmed by frontier orbital energy data obtained in electrochemical experiments (Supporting Information, Table Si). The term 'amorphous' used in all figures refers to a non-crystalline thin layer prepared by spin coating the sample solutions onto glass without any PMMA matrix. The term 'crystalline' used in all figures refers to ground single crystal samples loaded on filter paper.

Before discussing the changes in photophysical properties with concentration for the PDI compounds, it is useful to consider their molecular structure. The imide substitu- ents for the PDI compounds consisted of 2,5-substitutions on the 4-butylphenyl unit (Figure 1). Using bPDI-1 as reference, the size of the imide groups increased from phenyl to 3,5-di-tert-butylphenyl to 4-trityl for bPDI-2, bPDI3 and bPDI-4, respectively. From both molecular models (Figure $\mathrm{S}_{5}$ ) and single crystal X-ray structure data (Figures 3 to 5), it was evident that the imide substituents provided coverage of the perylene chromophore without perturbation of the planar aromatic core. The perylene $\pi$ surface of reference compound bPDI-1 is totally exposed ( $\sim 11 \AA$ ) while there is little space for intermolecular $\pi-\pi$ association for bPDI-3 and bPDI-4. In the crystal packing diagram, a staggered $\pi-\pi$ stacking arrangement was observed for bPDI-2 (Figure 3). This staggered arrangement has been observed previously in a liquid crystalline PDI derivative. ${ }^{10}$ Interestingly, no close $\pi-\pi$ contact between perylene cores was observed for in the crystal packing arrangement of bPDI-3 (Figure 4) while some perylene to perylene interactions were apparent for bPDI-4 (Figure 5). These observations will be considered and discussed in the context of the photophysical properties of the materials in the following sections.

a)
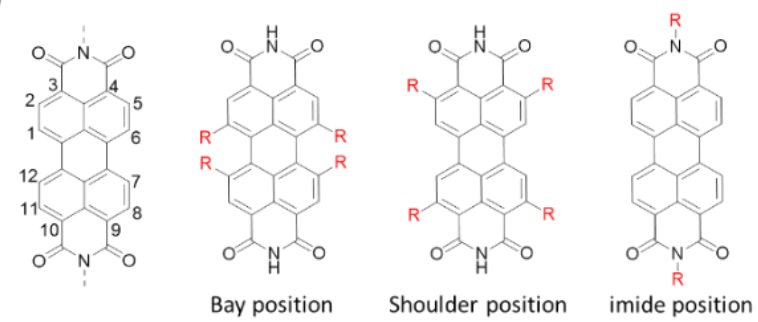

b)
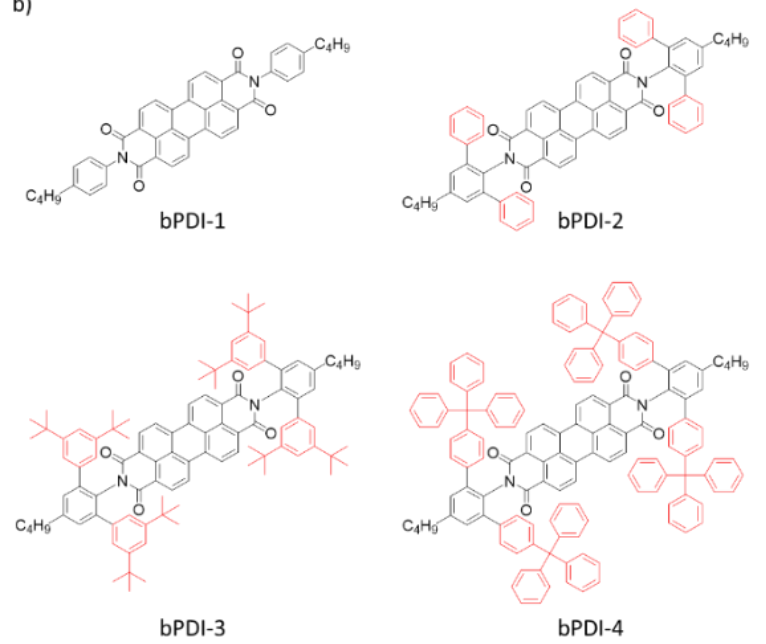

Figure 1. a) The three potential substituent positions on PDI type molecules and b) the structure of four bPDI compounds: bPDI-1, bPDI-2, bPDI-3 and bPDI-4.

\section{Reference compound bPDI-1}

Figure 3 shows the UV-vis absorption and PL spectrum of reference compound bPDI-1 in dilute solution $\left(2.5 \times 10^{-5}\right.$ $\mathrm{M}$ in $\mathrm{CHCl}_{3}$ ), dispersions in PMMA film and in an as-cast neat film. It has been observed previously for similar PDI derivatives, that aggregation resulting from intermolecu- 
lar $\pi-\pi$ association strongly affects the photophysical properties of the samples with increasing dye concentration. $^{25}$ At $10 \mathrm{mM}$ in the PMMA thin film, the absorption spectrum of bPDI-1 shows a significant blue-shift compared with the other conditions, along with a loss of vibronic features, indicative of $\mathrm{H}$-aggregation (Figure 3a). ${ }^{25}$ Upon increasing the concentration of bPDI-1 to $120 \mathrm{mM}$ in PMMA and in the neat amorphous thin-film, the absorption was more pronounced at lower energies and broader. The PL spectrum of bPDI-1 also varied with concentration (Figure $3 \mathrm{~b}$ ). The relative intensity of the vibronic band at $535 \mathrm{~nm}$ decreased with a concomitant emission at higher wavelengths, which may be attributed to emission from excited state aggregates. ${ }^{25}$ A marked decrease in the overall fluorescence intensity is also observed with increasing concentration (see below).

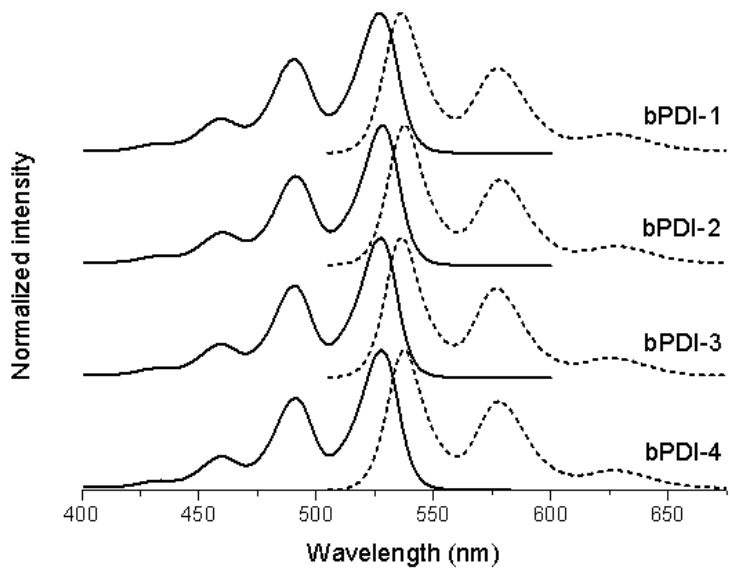

Figure 2. Absorption and emission spectra of all bPDIs in diluted solution.

\section{Phenyl-substituted bPDI-2}

The changes in photophysical properties with concentration for bPDI-2 (Figure 4) is very different to that of the reference compound bPDI-1. The aggregation of bPDI-2 is not $\mathrm{H}$-aggregate dominated as in the bPDI-1 material. Going from dilute solution to an amorphous neat film, slight shifts in the vibronic bands and broadening of the spectrum are observed. These changes can be attributed to a mixture of molecular aggregates including both $\mathrm{H}$ and J-aggregation. The changes in the PL spectrum of bPDI-2 are more pronounced compared with absorption spectrum changes (Figure 4). With increasing concentration in PMMA, a broad emission band developed with its maximum at $625 \mathrm{~nm}$, which can be assigned to molecular aggregates. Interestingly, the PL spectrum of the crystalline sample showed clear vibronic features while the amorphous neat sample only had one broad emission at $625 \mathrm{~nm}$. This indicated that molecular orientation and intermolecular association are very different in the two samples. It should be noted that polarization effects in different media may also contribute to small shifts in the absorption spectra. a)

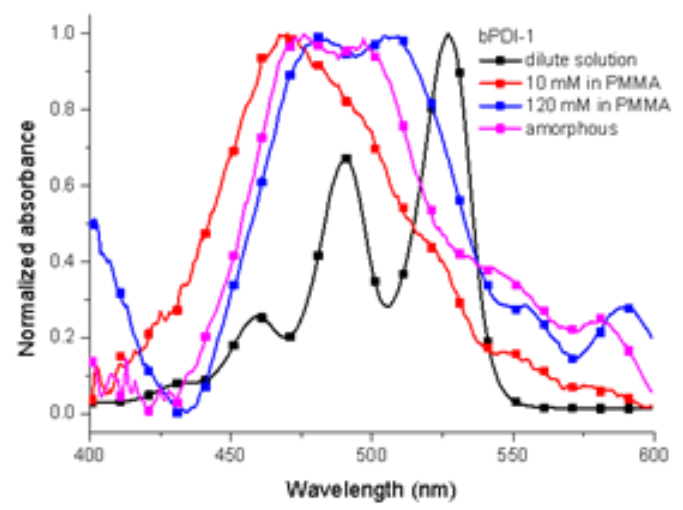

b)

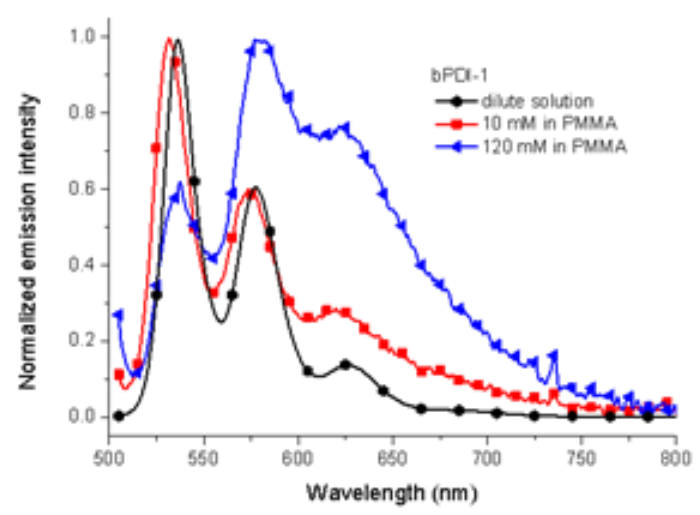

Figure 3. a) UV-vis absorption and b) PL spectra of bPDI-1 in various sample preparations.

\section{3,5-Di-tert-butylphenyl-substituted bPDI-3}

The UV-vis absorption spectra of bPDI- 3 in the various solution and solid state preparations were all similar, with clear vibronic features (Figure 5). This suggests that the bulky 3,5-di-tert-butylphenyl groups prevent close contact of the perylene chromophores, as intended. However, more marked changes in the PL spectra are observed but with a higher concentration tolerance of aggregationcaused quenching (ACQ) effect than bPDI-2. The dilute solution and $10 \mathrm{mM}$ PMMA spectra were essentially identical. A further increase in concentration in PMMA reveals the development of a red-shifted broad emission that dominated the amorphous neat film spectrum (Figure 5). The origin of the red-shifted broad emission in the neat bPDI-3 sample is unclear as the crystal structure data suggested little chance of close perylene-perylene interaction due to the steric bulk of the substituents. Similar to the bPDI-2 material, the crystalline bPDI-3 spectrum showed clear vibronic features while the amorphous neat sample exhibited only one broad emission feature. Again, these observations can be attributed to differences in molecular orientation and intermolecular association between the two samples. More intriguing was the observation that the PL spectrum of bPDI-2 and bPDI-3 crystalline samples were very similar in profile despite completely different crystal packing arrangements (Figures 4 and 5). 
a)
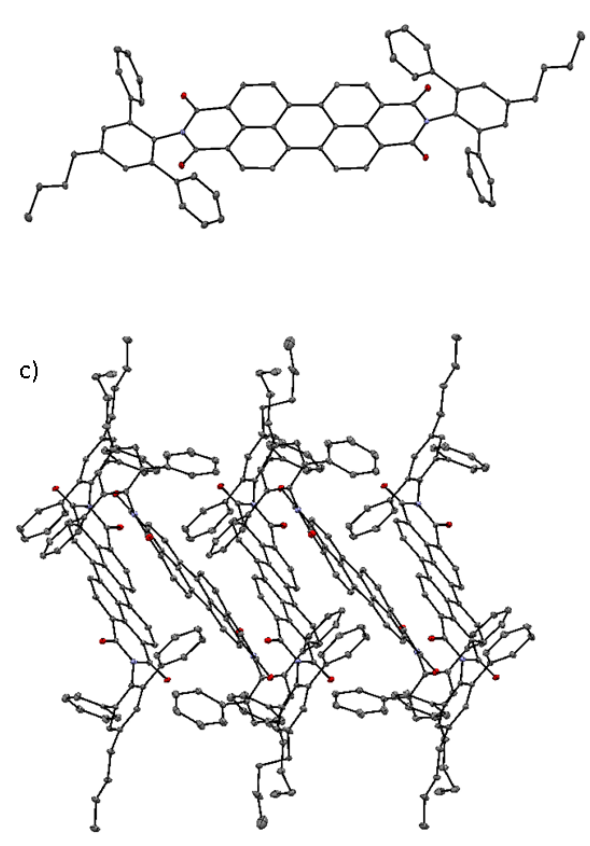

b)
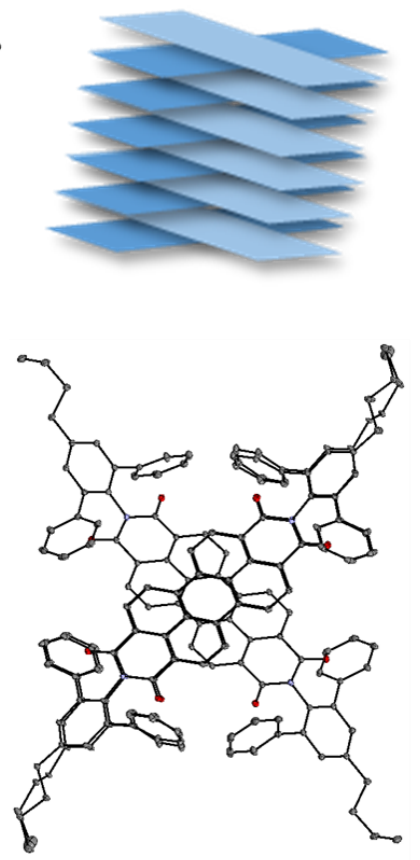

d)
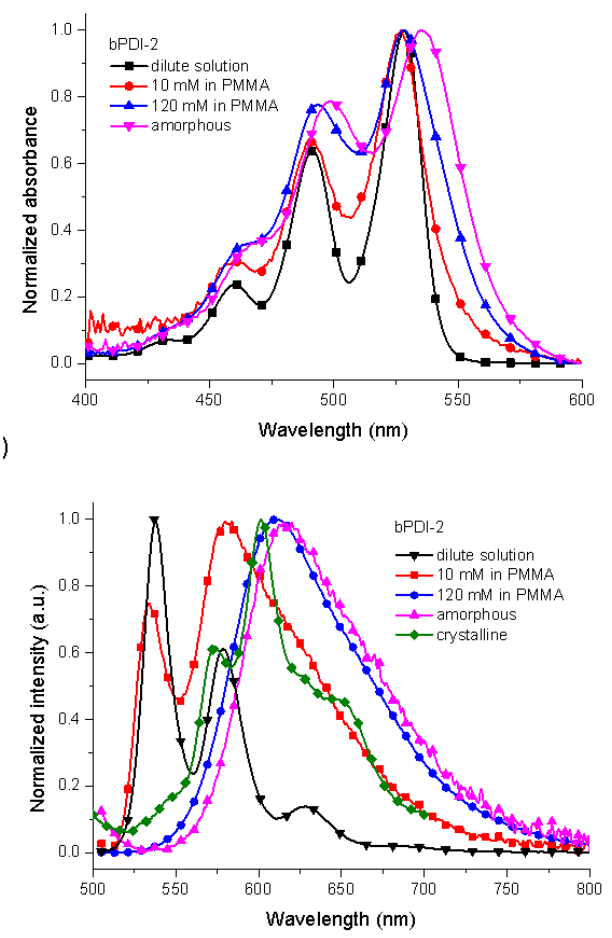

Figure 4. Representations of single crystal X-ray data of bPDI-2: a) the thermal ellipsoid illustration of one PDI unit; b) illustration of crystal packing pattern; c) the crystal packing diagram from side view and top view of $\pi$ - $\pi$ stacking and d) UV-vis absorption and e) PL spectra of bPDI-2 in various sample preparations.

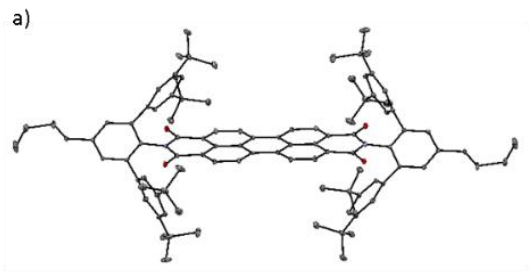

b)

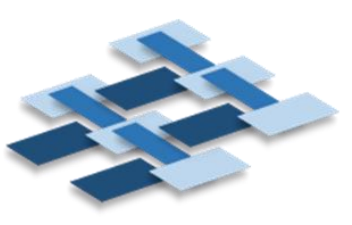

c)

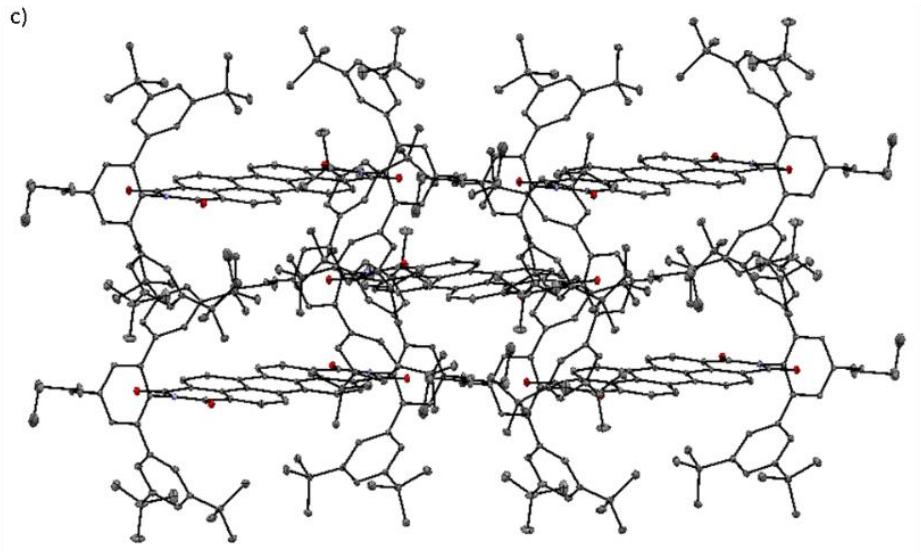

d)
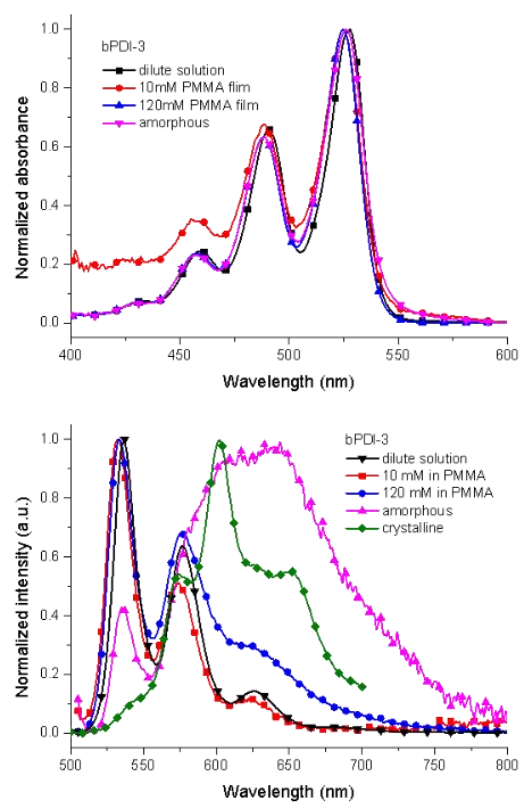

Figure 5. Representations of single crystal X-ray data of bPDI-3: a) the thermal ellipsoid illustration of one PDI unit; b) illustration of crystal packing pattern; c) the crystal packing diagram and d) UV-vis absorption and e) PL spectra of bPDI-3 in various sample preparations. 
a)

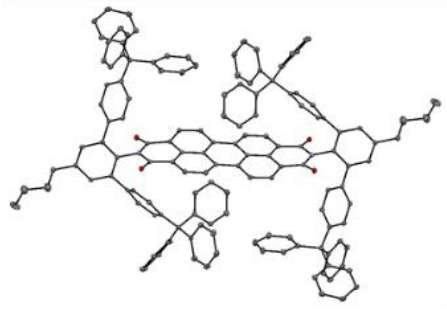

c)

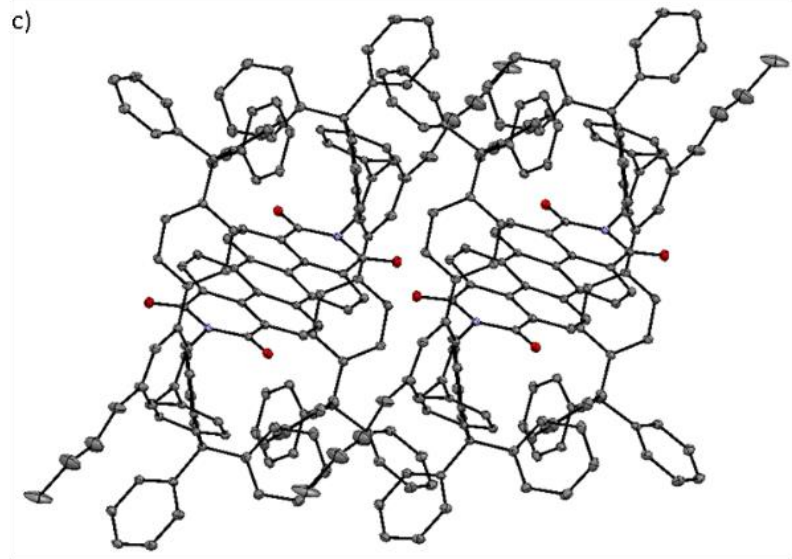

d)
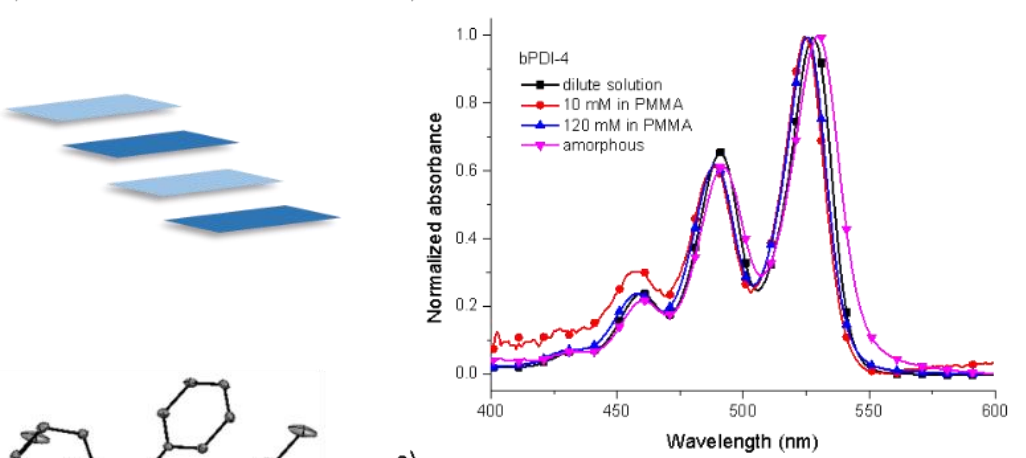

e)

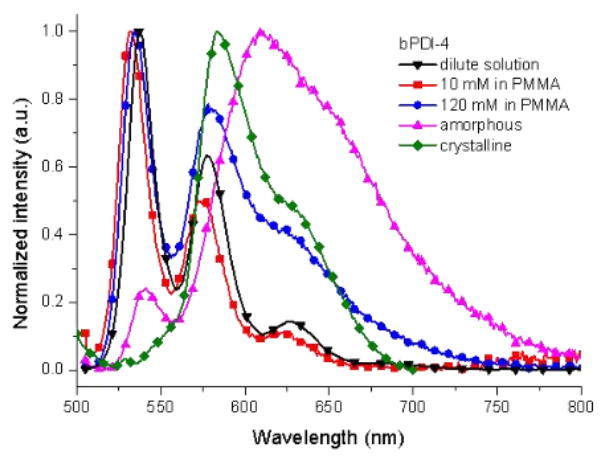

Figure 6. Representations of single crystal X-ray data of bPDI-4: a) the thermal ellipsoid illustration of one PDI unit; b) illustration of crystal packing pattern; c) the crystal packing diagram and d) UV-vis absorption and e) PL spectra of bPDI-4 in various sample preparations.

\section{4-Trityl-substituted bPDI-4}

The UV-vis absorption spectra of bPDI-4 in the various solution and solid state preparations were all very similar to one another in profile, with clear vibronic features (Figure 6). A small $3 \mathrm{~nm}$ red-shift of the absorption maximum was recorded when comparing the dilute solution sample to the neat film sample. The crystal packing data indicate the bulky trityl groups sit directly above and below the $\pi$ surface of the perylene core and some $\pi-\pi$ interaction are possible on the edges of the molecules (3.38 $\AA$ ). The slight red-shift in absorption spectrum might be related to polarization effect in the solid state (Figure 6d). As with bPDI-3, the PL spectra of bPDI-4 showed the development of a red-shifted emission with increasing concentration to the neat film indicating the presence of excited state aggregates (Figure 6). A large difference is observed in the PL spectra of the crystalline and amorphous samples again indicative of different molecular arrangement and packing in these samples.

To summarise, the strategy of using sterically bulky groups to prevent close contact of the perylene core chromophores was successful. Less predictable was the excited state properties as observed in the PL spectrum variations. For bPDI-2, 3 and 4, the PL spectrum showed the emergence of a red-shifted broad emission with increasing concentration in PMMA but remained emissive (high PLQY) at high concentration. This was in contrast with the reference compound bPDI-1 which showed significant quenching of emission with increasing concentration. The fluorescence decay profiles of bPDI- 3 and 4 were recorded at the low wavelength emission band as a function of concentration. These decays cannot be meaningfully interpreted in terms of either discrete decay components, or by lifetime distribution analyses. Instead, we have calculated the mean fluorescence lifetimes $\left(\tau_{\mathrm{m}}=\mathrm{a}_{1} \tau_{1}\right.$ $+\mathrm{a}_{2} \tau_{2}+. .+\mathrm{a}_{\mathrm{i}} \tau_{1}$, where $\left.\mathrm{a}_{1}+\mathrm{a}_{2}+. .+\mathrm{a}_{\mathrm{i}}=1\right)$ as a function of concentration. The $\tau_{\mathrm{m}} \mathrm{s}$ of these samples were smaller than that of the PDI monomer emission and decrease with increasing concentration (Figure $\mathrm{S}_{7}$ ). This indicates the presence of emissive excited state aggregates in concentrated samples. With consideration of the data discussed thus far, in the next section we discuss the concentration dependence of the PLQY of the PDI compounds in PMMA.

\section{PLQY of PDI derivatives}

It is clear from the above PL studies that bPDI-2, 3 and 4 are highly emissive at elevated concentration compared to the reference bPDI-1. To quantify the differences, the absolute PLQY of the PDI series dispersed in PMMA at a range of concentrations was measured using an integrating sphere. It is important to note that determining the absolute PLQY measurements, particularly for concentrated samples, were not straightforward. With significant overlap of the absorption and emission spectra for some 
of these PDI samples, a reabsorption correction must be applied to achieve the true PLQY of the chromophore (see SI for details). Figure 7 summarises the PLQY values for the four PDI compounds over concentrations ranging from 10 to $120 \mathrm{mM}$ in PMMA.

As expected, the PLQY of the reference compound bPDI-1 was only $21.0+0.9 \%$ at $10 \mathrm{mM}$ in PMMA which was much lower than the near unity value obtained from typical PDI compounds in dilute solution. ${ }^{4}$ This low PLQY is most likely the result of aggregation quenching given the strong intermolecular $\pi-\pi$ association in such PDI compounds as discussed earlier. On the other hand, the PLQY of bPDI-2 to 4 were above $90 \%$ at $10 \mathrm{mM}$ in PMMA with bPDI-4 being the highest at 100\% (Figure 7). A high PLQY was maintained up to $40 \mathrm{mM}$ with the values dropping to $80 \%, 46 \%$ and $72 \%$ at $120 \mathrm{mM}$ for bPDI 2,3 and 4 respectively. As neat films, the PLQY values for bPDI-2, 3 and 4 were $21 \%, 14 \%$ and $29 \%$ respectively, which are amongst the highest neat solid state PLQY of PDI-type materials reported. ${ }^{26-28}$

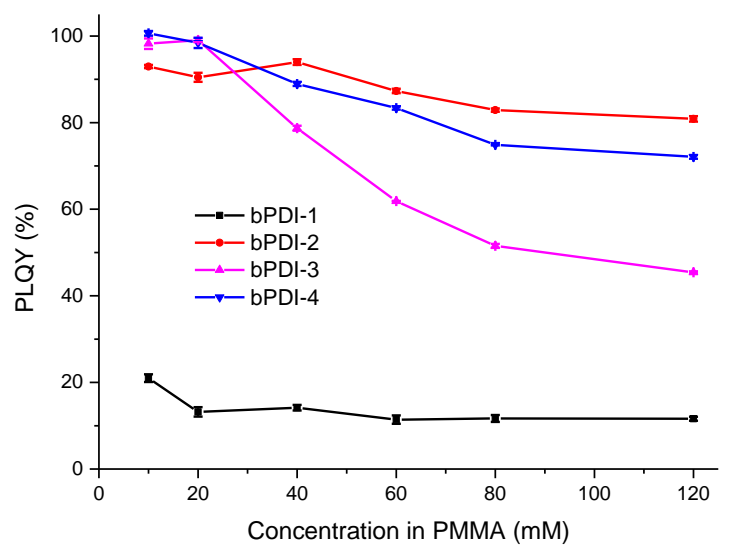

Figure 7. Absolute PLQY of the bPDI derivatives as a function of concentration in PMMA thin films. Reabsorption correction was applied to all data in this figure. Details of the PLQY measurement and the re-absorption correction can be found in Supporting Information. ${ }^{29}$

Three main contributions to these PLQY values can be considered: 1. Individual (monomeric) dye emission; 2. aggregate emission and 3. aggregation-caused quenching. Aggregation caused quenching is the dominant effect for the bPDI-1 reference compound. ${ }^{11}$ For bPDI-2, chromophore aggregation is evident in the absorption spectrum (Figure 4) but the aggregates remain emissive. At $120 \mathrm{mM}$ in PMMA, the PL spectrum of bPDI-2 consists of the redshifted broad emission with PLQY measured at $80 \%$, which is the highest of the bPDI series at this concentration. Interestingly the PLQY of bPDI-2 even slightly increased after the ground-state aggregation at $40 \mathrm{mM}$. In comparison, the PL of both bPDI- 3 and bPDI-4 involves a significant component of individual PDI emission up to very high concentrations (Figures 4 and 5). However, the PLQY decreases more markedly with concentration for bPDI-3 and 4 compared to bPDI-2. This observation sug- gests that excited state aggregates exist in concentrated samples of bPDI- 3 and 4 that are non-emissive despite the separation of the perylene core chromophore by the bulky di-tert-butylphenyl and trityl groups. While some of the variations in PLQY were unexpected, the concept of using bulky substituents to maintain the photophysical properties of the perylene chromophore with increasing concentration was successful. The trityl-substituted bPDI-4 shows typical PDI chromophore emission and relatively high PLQY at $120 \mathrm{mM}$ in PMMA out-performing the ditert-butylphenyl bPDI-3. The energy migration characteristics of bPDI-4 were then investigated and the results discussed below. Using an energy migration and energy trapping approach, the optical quantum efficiency (OQE, definition available in SI) of LSC devices containing bPDI4 as a FRET donor was compared with devices containing bPDI-3. ${ }^{12}$

\section{Energy migration and trapping}

In our previous work, an energy migration and trapping strategy was applied to improve the light harvesting performance of PDI-based LSC devices. ${ }^{12}$ The di-tertbutylphenyl bPDI-3 was used as an energy migration material and FRET donor while the commercial PDI dye Lumogen $^{\circledR}$ F Red 305 (LR305) was incorporated as the energy trap or FRET acceptor. The same spectroscopic experiments as described previously 3 were adopted to examine the trityl-substituted bPDI-4 in this work to compare its performance with bPDI-3.

In energy migration, the excitation energy residing in chromophores can undergo transfer between neighbouring molecules of the same type via a FRET process ${ }^{30}$, where the energy migration distance $\left(L_{D}\right)$ is dependent on the concentration of the dye. Apart from concentration, $L_{D}$ will depend on the FRET critical radius $\left(R_{0}\right)$, the separation at which energy transfer between chromophores and other intramolecular excited state relaxation processes are equally probable. ${ }^{31}$ A good overlap between the UVvis absorption and PL spectrum of the material is essential for an effective $R_{0}$ and an extended $L_{D}$. Given the spectral properties of bPDI-3 and bPDI-4, both chromophores are suitable for energy migration.

Using the spectral overlap and PLQY data and the Förster equation, the $R_{0}$ of homo-transfer within bPDI-3 and bPDI-4 was calculated to be 5.02 and $5.03 \mathrm{~nm}$ respectively (see SI for details). As with our previous study, energy migration in the PDI materials was further investigated using time-resolved fluorescence anisotropy. ${ }^{12}$ This technique enabled an alternative determination of $R_{0}$ through a Green's function model (see earlier work ${ }^{3}$ and SI for details). A $R_{0}$ value of $5.0 \mathrm{~nm}$ was obtained for both bPDI-3 and bPDI-4, which is in excellent agreement with the values calculated from the Förster equation. The migration distances, $L_{D}$, were also obtained from the time-resolved emission anisotropy measurements (see SI for detailed data). At $60 \mathrm{mM}$ in PMMA, the $L_{D}$ of bPDI- 3 and bPDI- 4 were determined to be 23 and $21 \mathrm{~nm}$ respectively. The $L_{D}$ value was greater than the average intermolecular dis- 
tance $(d)$ when the concentration exceeded $4 \mathrm{mM}$ (Figure $\mathrm{S}_{15 \mathrm{c}}$ ). At $120 \mathrm{mM}$, the $L_{D}$ of bPDI-4 reached $35 \mathrm{~nm}$, which is 30 times larger than the average intermolecular distance at this concentration. These results indicate that bPDI-3 and 4 are equally efficient for energy migration.

PMMA films containing a FRET donor, bPDI-4, and a FRET acceptor, $\mathrm{LR}_{305}$, were examined to determine the energy migration and trapping efficiency (Figure 9). LR305 should be an appropriate FRET acceptor for bPDI-4 as there is very good overlap between the PL spectrum of bPDI-4 and the absorption spectrum of LR305 (Figure 8a). The fluorescence quenching of the FRET donor by LR305 was investigated at two concentrations of donor - 60 and $120 \mathrm{mM}$ in PMMA. The fluorescence of the bPDI donor at either concentration was largely quenched by LR305 at 4 $\mathrm{mM}$ and completely quenched at $16 \mathrm{mM}$ (Figure S9). The samples in neat films (in the absence of the PMMA matrix), the emission of both bPDI-3 and bPDI-4 was effectively completely quenched by $1.7 \mathrm{~mol} \%$ of LR305 (Figure S9). The improvement of the trapping efficiency in neat films I s attributed to the increase in $L_{D}$ in these neat samples. and preferential trapping by the $\mathrm{LR}_{30} 5$.

a)

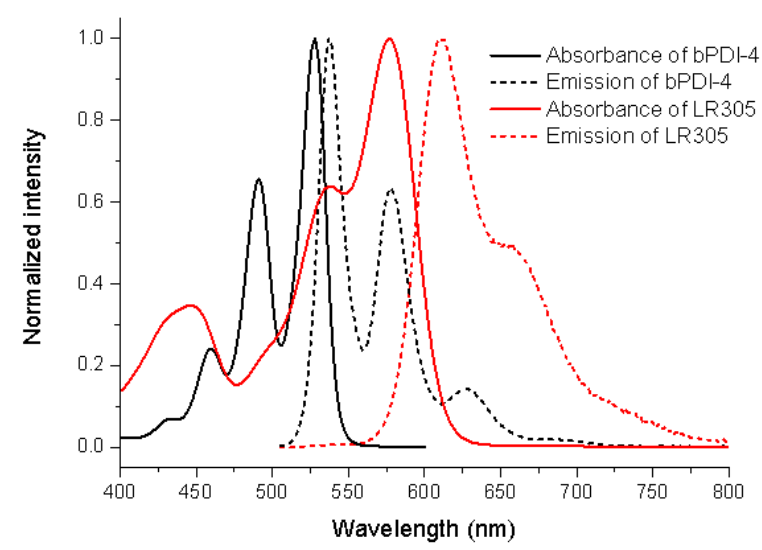

c)

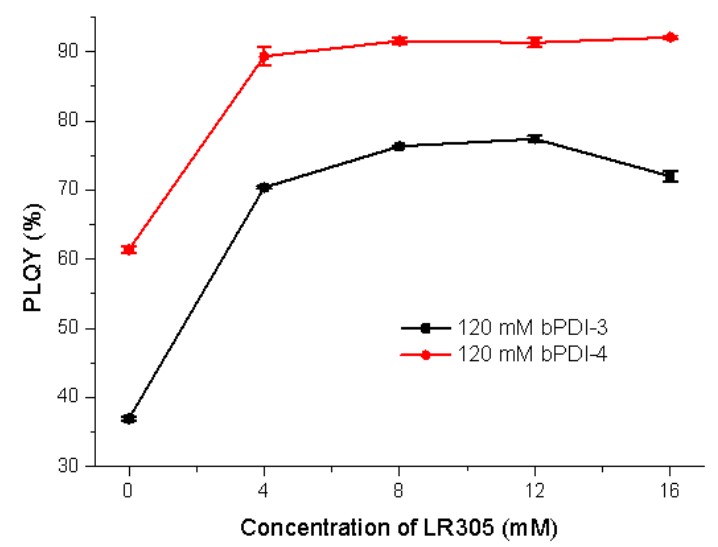

The PLQYs of these samples indicate that the addition of $\mathrm{LR}_{30} 5$ to the bPDI donors substantially improved the PLQY value (Figure 8b, c \& d). At $4 \mathrm{mM}$ of LR305, the overall PLQY was close to $90 \%$ for both bPDI donors at 60 $\mathrm{mM}$ while 90\% PLQY was observed for bPDI-4 at $120 \mathrm{mM}$. Even in neat films, the PLQY of bPDI-4 improved from $25 \%$ to $40 \%$ with the addition of $1.7 \mathrm{~mol} \%$ of LR305. These improvements in PLQY on addition of LR305 can be explained by the proposed energy migration and trapping process (Figure 9). In the samples containing bPDI compounds only, the excitation of the sample results in energy migration and emission from isolated PDI chromophores and/or aggregates depending on the concentration of the dye. As the concentration of PDI is increased, the PLQY value declines with the emergence of the aggregate emission as discussed earlier. By adding LR305, the excitation energy is ultimately trapped by the lower energy LR305 chromophore which exhibits high emission efficiency at the 4 to $16 \mathrm{mM}$ concentration range (Figure 8).

b)

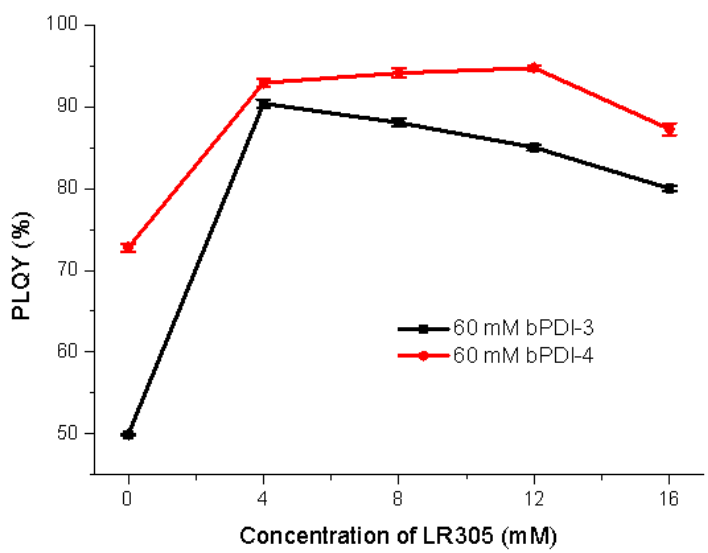

d)

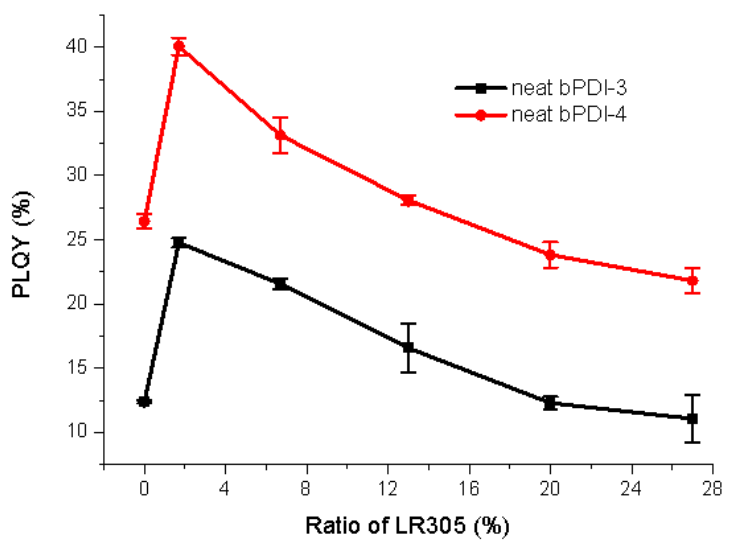

Figure 8. a) UV-vis absorption and PL spectra of bPDI-4 and LR305. The PLQY variation of the donor-acceptor system against the concentration of LR305 in: b) $60 \mathrm{mM}$ of the donors in PMMA; c) $120 \mathrm{mM}$ of the donors in PMMA and d) amorphous neat films of the donors. All PLQY data here is presented without the re-absorption correction, therefore the PLQY in o $\mathrm{mM}$ does not match the data in Figure 7. 
a)

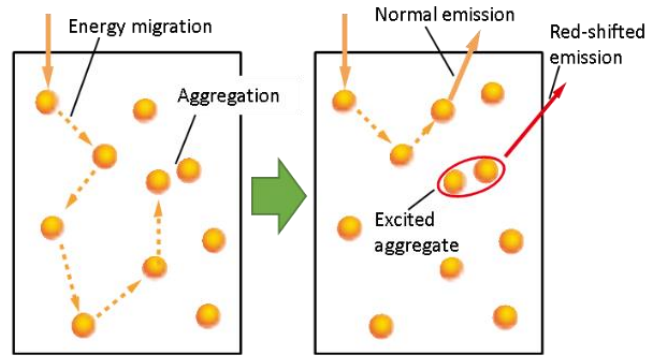

b)

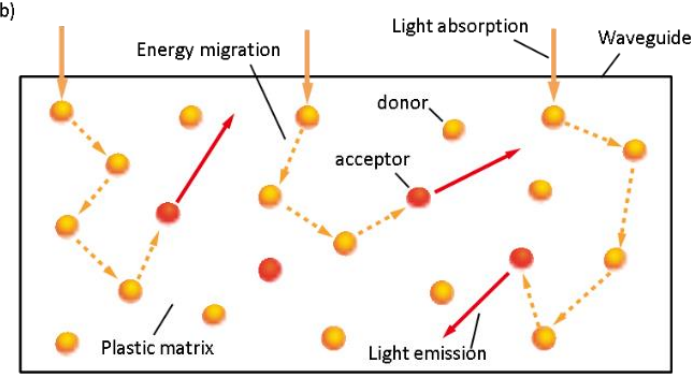

Figure 9. Proposed energy migration process for bPDI-3 and bPDI-4: a) energy migration followed by trapping by aggregates; b) the energy migration process followed by trapping by an acceptor.

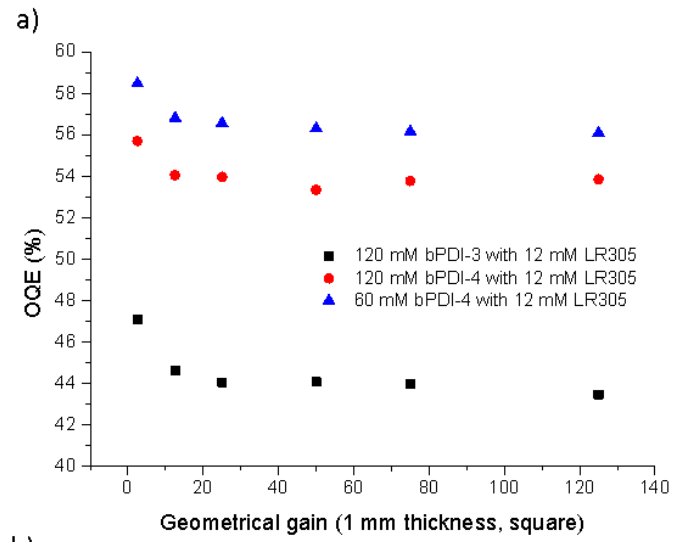

b)

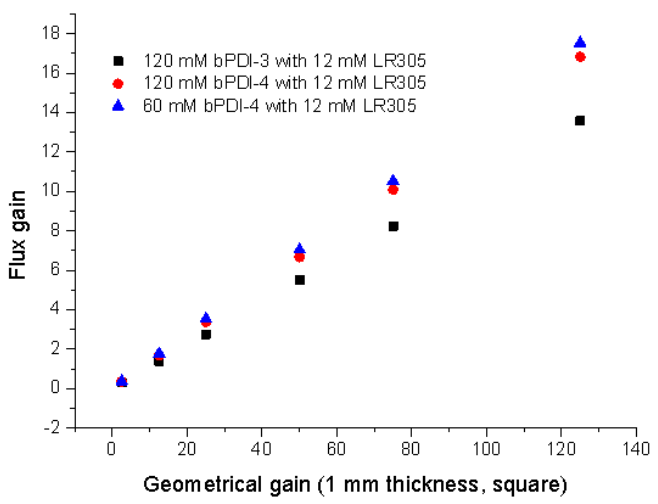

Figure 10. Simulated a) optical quantum efficiency (OQE) and b) flux gain of LSC devices based on $120 \mathrm{mM} \mathrm{bPDI}-4$ and bPDI-3 with $12 \mathrm{mM} \mathrm{LR} 305$ plotted as a function of the geometric gain on a $1 \mathrm{~mm}$ thick square glass waveguide. All samples were excited at $490 \mathrm{~nm}$. The absorbance of all samples was set to 1.0 at $490 \mathrm{~nm}$ and a total of 100,000 photons were used in each simulation.
Apart from high PLQY values, the use of these FRET donor-acceptor dye systems addresses the reabsorption issue that is detrimental to the performance of LSC devices. Monte-Carlo ray tracing simulation was performed to predict the performance of the LSCs devices based on bPDI-4 and bPDI-3 with 12 mM LR305 (Figure 10). Since the re-absorption of luminescence in all samples was small, the calculated OQE did not change significantly with increasing geometric gain, $G$ (Figure 10a, definitions of $\mathrm{OQE}$ and $\mathrm{G}$ in $\mathrm{SI}$ ). At $\mathrm{G}=12 \mathrm{O}$, the $\mathrm{OQE}$ of devices containing 60 and $120 \mathrm{mM}$ of bPDI- 4 were $56 \%$ and $54 \%$ respectively. The OQE of devices containing bPDI-3 at 120 $\mathrm{mM}$ was significantly lower than that of bPDI-4 primarily because of its lower PLQY value. It is noteworthy that the use of the new trityl bPDI-4 dye would result in 50\% reduction in the amount of LR305 in the polymer waveguide without compromising the light harvesting device performance. The flux gain of LSC devices containing bPDI-4 and $\mathrm{LR}_{30} 5$ at $\mathrm{G}=45$ was around 6 which was comparable to the performance of reported benchmark devices (Figure 1ob, definition of flux gain in SI). ${ }^{12,32,33}$

\section{Conclusion}

A series of molecularly-insulated perylene diimide derivatives were synthesized and fully characterized. By adding bulky substituents to the imide position, the intermolecular interactions of PDI chromophores were modulated, while the optical properties of the perylene core persisted. In contrast to the reference compound bPDI-1, that exhibits typical aggregation caused quenching of fluorescence with increasing concentration, emissive aggregates were observed for the phenyl-substituted bPDI-2. With the $\pi-\pi$ stacked crystal packing, bPDI-2 is an interesting material for organic light emitting devices which is currently under investigation in our group. The inter-chromophore interactions were further reduced for the di-tert-butylphenylsubstituted bPDI- 3 and the trityl-substituted bPDI-4 leading to isolation of the perylene core, as evident in their UV-vis absorption spectra. While bPDI-3 and bPDI-4 remained highly emissive at high concentrations in PMMA, a red-shifted broad emission became more prominent with increasing concentration. This suggested the presence of excited state aggregate species even though, from crystal structures, direct $\pi-\pi$ interactions between PDI chromophores must be small. Efficient energy migration was demonstrated in PMMA films containing bPDI- 3 and bPDI-4 at high concentrations by time-resolved fluorescence anisotropy experiments with an energy migration distance $\left(L_{D}\right)$ of $35 \mathrm{~nm}$ measured for bPDI-4 at $120 \mathrm{mM}$. Using bPDI-4 (120mM) as the FRET donor and LR305 $(4 \mathrm{mM})$ as the FRET acceptor in PMMA films, a PLQY of 90\% was obtained. With such a high PLQY and reduced re-absorption in this donor-acceptor dye system, light harvesting LSC devices were investigated giving an optical quantum efficiency of $54 \%$ and a flux gain of 6 at geometric gain of 45 . This light harvesting performance is comparable to the state-of-the-art LSCs, and we anticipate 
that these materials that maintain their high luminescence at high concentrations may find broad applications in large area devices especially on glass.

\section{ASSOCIATED CONTENT}

The Supporting Information is available free of charge on the ACS Publications website.

Synthesis and characterization of all new compounds, sample preparation for photophysical measurements, additional photophysical characterization data, calculations on energy migration, and Monte-Carlo ray tracing simulation.

\section{AUTHOR INFORMATION}

\section{Corresponding Author}

*wwhwong@unimelb.edu.au

\section{ORCID}

Wallace W. H. Wong: oooo-ooo1-7131-8532

Bolong Zhang: oooo-0002-2955-8409

\section{Funding Sources}

Australian Renewable Energy Agency

Australian Research Council (ARC)

Notes

The authors declare no competing financial interest.

\section{ACKNOWLEDGMENT}

This work was made possible by support from the Australian Renewable Energy Agency which funds the project grants within the Australian Centre for Advanced Photovoltaics (ACAP). Responsibility for the views, information or advice expressed herein is not accepted by the Australian Government. WWHW is supported by an ARC Future Fellowship (FT130100500). KPG, TAS and WWHW are also supported by the ARC Centre of Excellence in Exciton Science (CE170100026). We would like to thank Dr. James Banal for constructive discussions.

\section{REFERENCES}

1. $\quad$ Fan, Q.; Cheng, K.; Yang, Z.; Zhang, R.; Yang, M.; Hu, X.; Ma, X.; Bu, L.; Lu, X.; Xiong, X.; Huang, W.; Zhao, H.; Cheng, Z., Perylene - Diimide - Based Nanoparticles as Highly Efficient Photoacoustic Agents for Deep Brain Tumor Imaging in Living Mice. Adv. Mater. 2015, 27, 843-847.

2. Li, G.; Zhao, Y.; Li, J.; Cao, J.; Zhu, J.; Sun, X.; Zhang, Q., Synthesis, Characterization, Physical Properties, and OLED Application of Single BN-Fused Perylene Diimide. J. Org. Chem. 2014, 80, 196-203.

3. Briseno, A. L.; Mannsfeld, S. C. B.; Reese, C.; Hancock, J. M.; Xiong, Y.; Jenekhe, S. A.; Bao, Z.; Xia, Y., Perylenediimide Nanowires and Their Use in Fabricating Field-Effect Transistors and Complementary Inverters. Nano Lett. 2007, 7, 2847-2853.

4. Huang, C.; Barlow, S.; Marder, S. R., Perylene3,4,9,10-tetracarboxylic Acid Diimides: Synthesis, Physical Properties, and Use in Organic Electronics. J. Org. Chem. 2011, 76, 2386-2407.
5. $\quad$ Chen, W.; Yang, X.; Long, G.; Wan, X.; Chen, Y.; Zhang, Q., A Perylene Diimide (PDI)-based Small Molecule with Tetrahedral Configuration as a Non-fullerene Acceptor for Organic Solar Cells. J. Mater. Chem. C 2015, 3, 4698-4705.

6. Würthner, F.; Saha-Moller, C. R.; Fimmel, B.; Ogi, S.; Leowanawat, P.; Schmidt, D., Perylene Bisimide Dye Assemblies as Archetype Functional Supramolecular Materials. Chem. Rev. 2016, 116, 962-1052.

7. Kozma, E.; Catellani, M., Perylene Diimides based Materials for Organic Solar Cells. Dyes Pigm. 2013, 98, 160179 .

8. Yan, P.; Chowdhury, A.; Holman, M. W.; Adams, D. M., Self-organized Perylene Diimide Nanofibers. J. Phys. Chem. B 2005, 109, 724-730.

9. Ide, J.; Mereau, R.; Ducasse, L.; Castet, F.; Olivier, Y.; Martinelli, N.; Cornil, J.; Beljonne, D., Supramolecular Organization and Charge Transport Properties of Selfassembled $\pi-\pi$ Stacks of Perylene Diimide Dyes. J. Phys. Chem. B 2011, 115, 5593-603.

10. Chen, Z.; Stepanenko, V.; Dehm, V.; Prins, P.; Siebbeles, L. D.; Seibt, J.; Marquetand, P.; Engel, V.; Würthner, F., Photoluminescence and Conductivity of Selfassembled $\pi-\pi$ Stacks of Perylene Bisimide Dyes. Chem. Eur. J. 2007, 13, 436-449.

11. Mei, J.; Leung, N. L.; Kwok, R. T.; Lam, J. W.; Tang, B. Z., Aggregation-Induced Emission: Together We Shine, United We Soar! Chem. Rev. 2015, 115, 11718-940.

12. Banal, J. L.; Soleimaninejad, H.; Jradi, F. M.; Liu, M.; White, J. M.; Blakers, A. W.; Cooper, M. W.; Jones, D. J.; Ghiggino, K. P.; Marder, S. R.; Smith, T. A.; Wong, W. W. H., Energy Migration in Organic Solar Concentrators with a Molecularly Insulated Perylene Diimide. J. Phys. Chem. C 2016, 120, 12952-12958.

13. Osswald, P.; Würthner, F., Effects of Bay Substituents on the Racemization Barriers of Perylene Bisimides: Resolution of Atropo-enantiomers. J. Am. Chem. Soc. 2007, 129, 14319-14326.

14. Osswald, P.; Leusser, D.; Stalke, D.; Würthner, F., Perylene Bisimide based Macrocycles: Effective Probes for the Assessment of Conformational Effects on Optical Properties. Angew. Chem. Int. Ed. 2004, 44, 250-253.

15. Ramanan, C.; Smeigh, A. L.; Anthony, J. E.; Marks, T. J.; Wasielewski, M. R., Competition between Singlet Fission and Charge Separation in Solution-Processed Blend Films of 6,13-Bis(triisopropylsilylethynyl)pentacene with Sterically-Encumbered Perylene-3,4:9,10-bis(dicarboximide)s. J. Am. Chem. Soc. 2012, 134, 386-397.

16. Mitsui, M.; Fukui, H.; Takahashi, R.; Takakura, Y.; Mizukami, T., Single-Molecule Fluorescence Spectroscopy of Perylene Diimide Dyes in a gamma-Cyclodextrin Film: Manifestation of Photoinduced H-Atom Transfer via Higher Triplet (n, $\pi^{*}$ ) Excited States. J. Phys. Chem. A. 2017, 121, 15771586.

17. Takashima, Y.; Fukui, Y.; Otsubo, M.; Hamada, N.; Yamaguchi, H.; Yamamoto, H.; Harada, A., Emission Properties of Cyclodextrin Dimers Linked with Perylene Diimide - Effect of Cyclodextrin Tumbling. Polym. J. 2012, 44, 278-285.

18. Nakazono, S.; Easwaramoorthi, S.; Kim, D.; Shinokubo, H.; Osuka, A., Synthesis of Arylated Perylene Bisimides through $\mathrm{C}-\mathrm{H}$ Bond Cleavage under Ruthenium Catalysis. Org. Lett. 2009, 11, 5426-5429. 
19. Li, X.; Wang, H.; Schneider, J. A.; Wei, Z.; Lai, W.Y.; Huang, W.; Wudl, F.; Zheng, Y., Catalyst-free one-step synthesis of ortho-tetraaryl perylene diimides for efficient OPV non-fullerene acceptors. J. Mater. Chem. C 2017, 5, 27812785 .

20. Ma, Y.-S.; Wang, C.-H.; Zhao, Y.-J.; Yu, Y.; Han, C.X.; Qiu, X.-J.; Shi, Z., Perylene Diimide Dyes Aggregates: Optical Properties and Packing Behavior in Solution and Solid State. Supramol. Chem. 20o7, 19, 141-149.

21. Biedermann, F.; Elmalem, E.; Ghosh, I.; Nau, W. M.; Scherman, O. A., Strongly Fluorescent, Switchable Perylene Bis(diimide) Host-guest Complexes with Cucurbit[8]uril in Water. Angew. Chem. Int. Ed. 2012, 51, 7739-7743.

22. Herrmann, A.; Weil, T.; Sinigersky, V.; Wiesler, U. M.; Vosch, T.; Hofkens, J.; De Schryver, F. C.; Müllen, K., Polyphenylene Dendrimers with Perylene Diimide as a Luminescent Core. Chem. Eur. J. 2oo1, 7, 4844-4853.

23. Banal, J. L.; Ghiggino, K. P.; Wong, W. W., Efficient Light Harvesting of a Luminescent Solar Concentrator using Excitation Energy Transfer from an Aggregation-induced Emitter. Phys. Chem. Chem. Phys. 2014, 16, $25358-25363$.

24. $\quad$ Banal, J. L.; Zhang, B.; Jones, D. J.; Ghiggino, K. P.; Wong, W. W. H., Emissive Molecular Aggregates and Energy Migration in Luminescent Solar Concentrators. Acc. Chem. Res. 2017, 50, 49-57.

25. Haines, C.; Chen, M.; Ghiggino, K. P., The Effect of Perylene Diimide Aggregation on the Light Collection Efficiency of Luminescent Concentrators. Sol. Energy Mater. Sol. Cells 2012, 105, 287-292.

26. Lin, M.-J.; Jiménez, Á. J.; Burschka, C.; Würthner, F., Bay-substituted Perylene Bisimide Dye with an Undistorted
Planar Scaffold and Outstanding Solid State Fluorescence Properties. Chem. Commun. 2012, 48, 12050-12052.

27. Ramírez, M. G.; Pla, S.; Boj, P. G.; Villalvilla, J. M.; Quintana, J. A.; Díaz - García, M. A.; Fernández - Lázaro, F.; Sastre - Santos, Á., 1,7 - Bay - Substituted Perylenediimide Derivative with Outstanding Laser Performance. Advanced Optical Materials 2013, 1, 933-938.

28. Jiménez, Á. J.; Lin, M.-J.; Burschka, C.; Becker, J.; Settels, V.; Engels, B.; Würthner, F., Structure-Property Relationships for 1,7-Diphenoxy-perylene Bisimides in Solution and in the Solid State. Chem. Sci. 2o14, 5, 6o8-619.

29. Wilson, L. R.; Richards, B. S., Measurement Method for Photoluminescent Quantum Yields of Fluorescent Organic Dyes in Polymethyl Methacrylate for Luminescent Solar Concentrators. Appl. Opt. 2009, 48, 212-220.

3o. Olson, R. W.; Loring, R. F.; Fayer, M. D., Luminescent Solar Concentrators and the Reabsorption Problem. Appl. Opt. 1981, 20, 2934-2940.

31. Hildebrandt, N., How to Apply FRET: From Experimental Design to Data Analysis. In FRET - Förster Resonance Energy Transfer: From Theory to Applications, Wiley-VCH: 2013.

32. Erickson, C. S.; Bradshaw, L. R.; McDowall, S., Zeroreabsorption Doped-nanocrystal Luminescent Solar Concentrators. ACS Nano 2014, 8, 3461-3467.

33. Currie, M. J.; Mapel, J. K.; Heidel, T. D.; Goffri, S.; Baldo, M. A., High-efficiency Organic Solar Concentrators for Photovoltaics. Science 2008, 321, 226-228. 

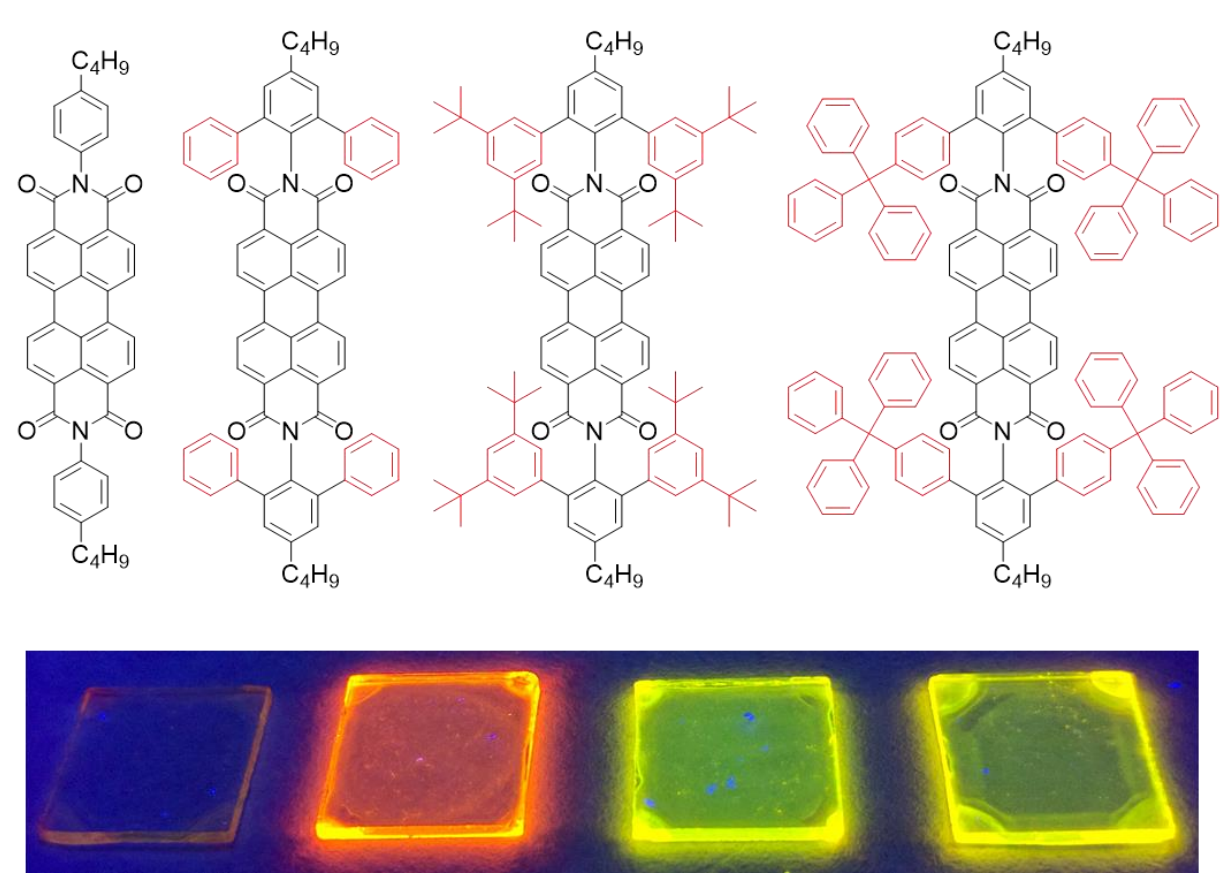